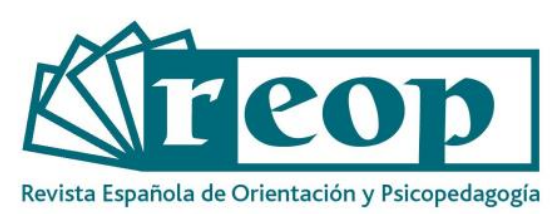

\title{
EFECTOS DE UN PROGRAMA DE INTERVENCIÓN SOBRE LAS HABILIDADES EMOCIONALES EN NIÑOS/AS PREESCOLARES
}

\section{EFFECTS OF AN INTERVENTION PROGRAM ON EMOTIONAL SKILLS IN PRESCHOOL CHILDREN}

\author{
Mirian Grimaldo Muchotrigo ${ }^{1}$ \\ Universidad Peruana de Ciencias Aplicadas. Oficina de Investigación Institucional. Monterrico- \\ Santiago de Surco, Perú
}

\section{César Merino-Soto}

Universidad San Martín de Porres. Lima, Perú

\section{RESUMEN}

El objetivo de este estudio es determinar los efectos del programa de intervención Descubriendo Mis emociones cuyo objetivo es reconocer y expresar las emociones positivas y morales, y el manejo de emociones negativas en un grupo de niños/as en edad preescolar de Lima. El estudio ha seguido un diseño de investigación cuasi experimental con grupo de control con pre test y post test. La muestra estuvo conformada por 125 participantes con una edad promedio de 5 años (95 del grupo experimental y 30 del control). Se determinó la evidencia basada en la validez de contenido, de constructo y confiabilidad del instrumento. El programa se organizó en diez sesiones semanales de aproximadamente dos horas cada una, mediante la aplicación de técnicas

${ }^{1}$ Correspondencia: Mirian Grimaldo. Universidad Peruana de Ciencias Aplicadas, Oficina de Investigación Institucional, Prolongación Primavera 2390, Monterrico, Santiago de Surco. Teléfono: 996401005. Correo-e: mirian.grimaldo@upc.pe 
participativas. Al inicio de la intervención, no se encontraron diferencias significativas en los puntajes obtenidos por los niños/as que conformaron el grupo control y el grupo experimental. En el análisis intragrupos, los dos grupos experimentales mostraron leves diferencias; en el análisis entre-grupos, se detectaron claras diferencias entre el grupo control y los grupos experimentales; en el análisis clinicométrico, hubo un mayor cambio confiable y clínico en los grupos experimentales. Se discuten las implicaciones de un programa orientado al reconocimiento de emociones en niños/as.

Palabras clave: Programa de intervención, educación inicial, emociones, infancia, educación emocional.

\section{ABSTRACT}

The goal of this study is to determine the effects of an intervention program discovering my Emotions for recognizing and expressing positive, negative and moral emotions. It was conducted with a group of preschooler's children in Lima. The study followed a quasi-experimental research design with pre-test and post-test control groups. The study sample comprised 125 participants with an average age of 5 years (95 in the experimental group and 30 in the control group), through intentional sample. Content validity, construct validity and reliability of the instrument were determined. The program was organized in ten weekly sessions of approximately two hours per session by applying participatory techniques. In the pretest, no significant differences were found in the scores obtained from the control group and the experimental group. In the intra-test group analysis, the two experimental groups showed slight differences. In the posttest, clear differences were detected between the control group and the experimental groups; in the clinical metric analysis, there was a greater reliable and clinical change in the experimental groups. The strategies, activities and participatory techniques are analysed, the regular attendance of the participants, among other aspects and the implications of the program oriented to the recognition of emotions in children are discussed. Pre-school children who participated in the program were able to recognize and express positive, moral and negative emotions. This demonstrates the effectiveness of the program and verifies the hypothesis formulated.

Key Words: Intervention program, preschool education, emotions, childhood, emotional education.

\section{Introducción}

Durante los primeros años de vida resulta necesario orientar las acciones educativas, incluso considerarlas como esenciales en el proceso de desarrollo (Rendón, 2007), especialmente las competencias emocionales (Gutiérrez, Escartí y Pascual, 2011), pues constituyen recursos para el desarrollo psicológico. Son beneficiosas porque favorecen el control de conductas agresivas (Mestre, Samper y Frías, 2002), facilitan un mejor sentido de vida (Hicks, Schlegel y King, 2010), estimulan el desarrollo del pensamiento creativo (Heinze, Miller, Seifer, Dickstein y Locke, 2015), permiten el bienestar (Ato, González y Carranza, 2004) y propician los logros académicos (Denham, Bassett, Brown, Way, y Steed, 2013). Son perjudiciales, cuando generan desregulación 
emocional (Hilt, Hanson y Pollak, 2011) o promueven irritabilidad (Fredrickson, 2018; Pérez y Guerra, 2014).

Por lo anterior, resulta crucial implementar un programa orientado a desarrollar habilidades emocionales en niños/as/as, de tal forma que les permita vivir de forma saludable, regulando sus emociones mediante estrategias orientadas a la flexibilidad emocional (Monin, Pizarro y Beer, 2007). Busca el desarrollo de competencias orientadas a la conciencia emocional (Bisquerra, 2005) y desde la perspectiva de Mayer y Salovey (1997), quienes plantean un modelo que abarca procesos psicológicos básicos (percepción, evaluación y expresión emocional) y complejos (regulación y promoción del crecimiento emocional e intelectual).

El reconocimiento de las emociones parece ser un aspecto central en la adaptación social de las personas en muchos rango de edad (Tarnowski, Kołodziej, Majkowski, y Rak, 2017). Se encuentra presente en el proceso de interacción social, pues brinda información acerca del estado afectivo de las personas (Rueda, 2017). Así, la expresión facial se centra principalmente en los ojos y la boca (Haxby, Hoffman y Gobbini, 2000), lo cual se enfatiza en la presentación, si es de frente o de perfil y la configuración, mirada y posición de la comisura de los labios (Bruce y Yong, 1986). Se implican varias áreas del encéfalo, así la mirada está relacionada con el surco intraparietal (Hoffman y Haxby, 2000) y la amígdala, es responsable del procesamiento del contenido de las emociones (Saavedra, Díaz, Zúñiga, Navia y Zamora, 2015).

Para el caso de la clasificación de las emociones positivas, el presente estudio se basa en el modelo de Fredrickson (1998), quien plantea que las emociones positivas son predictores que desencadenan espirales ascendentes al bienestar emocional (Catalino y Fredrickson, 2011; Fredrickson, Mancuso, Branigam, y Tugade, 2000; Fredrickson y Joiner, 2002; Fredrickson, 2013; Oros, 2014). Desde esta perspectiva, las emociones positivas promueven estrategias cognitivas específicas y aumentan los recursos intelectuales, proporcionan niveles altos de rendimiento académico, facilitan el entendimiento de las situaciones complejas (Catalino y Fredrickson, 2011) y hacen posible la creación de relaciones sociales (Ccaypani, 2011).

En cuanto a las emociones negativas, se conoce que cuando son intensas y experimentadas habitualmente, impactan en la calidad de vida y bienestar de las personas (Piqueras, Ramos, Martínez y Oblitas, 2009). Constituyen uno de los principales factores de riesgo para contraer enfermedades físicas y mentales (Oblitas, 2008), podrían generar altos grados de malestar (Pulido y Herrera, 2017; Pulido y Herrera; 2015; Kushnir, Gothelf y Sadeh, 2014), se relacionan con el estrés (Oblitas, 2008) y producen ideas negativas (Adler, 2017).

En el desarrollo afectivo, las emociones positivas y las negativas se complementan (Fredrickson, 2001) y ambas son importante en el desarrollo evolutivo (Vecina, 2006). Por ejemplo, las emociones negativas permiten que el ser humano se pueda enfrentar a situaciones de supervivencia (Piqueras, et al., 2009), frente al miedo surge la función de protección y frente a la ira se genera la destrucción (Chóliz, 2005). Respecto a las emociones morales, se pensaba que la moral implicaba principalmente el estudio del razonamiento moral (Kohlberg, 1984). Sin embargo, en la actualidad se considera que las emociones están presentes en la moral (Haidt, 2007).

En cuanto a los estudios de intervención se han realizado investigaciones con el objetivo de mejorar las prácticas de socialización de las emociones en padres/madres de niños/as en edad preescolar (Havighurst, Wilson, Harley y Prior, 2009), mediante programas de crianza centrados en la emoción de los padres/madres de niños/as pequeños (Lauw, Havighurst, Wilson, Harley y Northam, 2014), con el propósito de entrenar habilidades emocionales, para que a su vez los niños/as mejoren sus relaciones y por tanto su conducta. Aunque también se encuentran estudios de intervención realizados con niños/as a nivel preescolar (Amesty y Clinton, 2009), niños/as de primaria (Ambrona, López-Pérez y Márquez-González; 2012; Celdrán y Ferrándiz, 2012) y en niños/as y adolescentes (Obiols, 2005). A partir de lo planteado se formula la siguiente hipótesis: 
el programa de intervención es eficaz para el reconocimiento y expresión de emociones positivas, negativas y morales en un grupo de niños/as en edad preescolar.

El presente estudio tiene por objetivo evaluar la aplicación de un programa orientado al reconocimiento de emociones positivas y morales, y del manejo de las emociones negativas. Debido a que se realiza en el mismo ambiente escolar de los niños/as participantes, los resultados tendrán particular importancia al producir resultados esperados en un ambiente en que concurren varias influencias para promover o retener los efectos del programa.

Debido a que la evaluación de los cambios estimados con estadísticos de grupo, pueden enmascarar la variabilidad individual del cambio, se aplicó también la metodología clinicométrica, para estimar el cambio individual. En el presente estudio, el efecto de la intervención se analizó mediante la significancia del cambio individual, dentro del concepto de cambio clínicamente significativo (Bauer, Lambert, y Nielsen, 2004), referido como la magnitud del cambio que tiene importancia clínica para la persona sujeta a intervención. Para implementarlo, existen varios métodos disponibles, pero la literatura relevante ha demostrado la tendencia hacia la convergencia entre los métodos (Atkins, Bedics, McGlinchey, y Beauchaine, 2005; Bauer, Lambert, y Nielsen, 2004; Beurs et al., 2015; Hafkenscheid, 2000; McGlinchey, Atkins, y Jacobson, 2002; Ronk, Hooke, y Page, 2012; Ronk, Hooke y Page, 2016). Aunque esta convergencia varía de acuerdo al dominio conductual, las características de los instrumentos utilizados, y la incorporación de ajustes por regresión a la media, hay una clara tendencia para elegir un método parsimonioso y eficiente (Beurs et al., 2015; Ronk et al., 2012; Ronk et al., 2016; Wise, 2004), como aquel identificado como el enfoque clásico (Bauer et al., 2004), es decir, el método de Jacobson y Truax (JT, 1991).

\section{Método}

\section{Diseño y muestra}

Se aplicó un diseño de investigación cuasi experimental con grupo control con pre test y post test (Alarcón, 2008).

El muestreo seleccionado para la presente investigación, fue no probabilístico e intencional. Las instituciones educativas elegidas reciben a niños/as de familias que residen en la misma localidad y comparten similares características demográficas y socioeconómicas. La matrícula a estos colegios es libre y universal, y no hay un proceso de selección, por ello se consideró que la distribución de población de niños/as en las instituciones no presenta un sesgo relevante para los resultados. Las instituciones no cuentan con servicio psicológico, y no se realizaron previamente talleres relacionados con los constructos investigados.

La muestra efectiva estuvo conformada por 125 participantes, 95 (76\%) del grupo experimental y $30(24 \%)$ del grupo control. Niños/as/as con una edad promedio de 5 años, entre varones $(41.6 \%)$ y mujeres $(58.4 \%)$ del turno mañana $(61.6 \%)$ y tarde $(38.4 \%)$, de tres centros educativos estatales seleccionados intencionalmente. Respecto a las características familiares, $63.2 \%$ provienen de familias nucleares y $36.8 \%$ de familias extensas. 
El principal criterio de inclusión de la muestra fue la asistencia regular a clases. Se excluyeron a niños/as/as cuyos puntajes en la entrevista fueron elevados o por inasistencia a dos o más sesiones. El criterio de elevado puntaje en el pre-test indicaba que el niño/a tendía a una alta habilidad en el reconocimiento de emociones y produciría un efecto de la distribución de los puntajes.

El instrumento fue aplicado por un equipo de cinco estudiantes de los últimos ciclos de la carrera de psicología de una Universidad Particular de Lima, tres mujeres y dos varones, con una edad promedio de 21 años, un promedio de 18 créditos matriculados y un promedio ponderado de 15.5 en adelante, lo cual indicaba un buen rendimiento.

\section{Instrumentos}

Se construyó una Lista de Chequeo observacional, que contenía una lista de diez emociones, evaluadas mediante dos ítems en cada una. Consta de 20 ítems, divididos en tres subescalas: emociones positivas (8 ítems), emociones negativas (6 ítems) y emociones morales (6 ítems). Es de fácil administración y tiene una duración aproximada entre 10 a 15 minutos. En cuanto a la validez del instrumento, se verificó examinando el contenido mediante la consulta a cinco investigadores y psicólogos clínicos, especialistas en psicología infantil, dos con el grado de doctor y tres magisters, con una experiencia aproximada de 5 años; cuyo juicio sirvió para comprobar lo apropiado del contenido en relación con los objetivos de la entrevista. Los resultados se cuantificaron con el coeficiente $V$ (Aiken, 1980, como se citó en Escurra, 1988) y se obtuvieron valores altos $(V>.90)$ para cada ítem (Tabla 1$)$.

\section{Tabla 1}

Estructura interna: una y dos dimensiones

\begin{tabular}{lccc} 
& Unidimensional & \multicolumn{2}{c}{ Dos dimensiones } \\
& $\mathbf{F}$ & $\mathbf{F 1}$ & $\mathbf{F 2}$ \\
\hline Alegría & $.547^{*}$ & .183 & $.481^{*}$ \\
Interés & $.398^{*}$ & $.636^{*}$ & .004 \\
Satisfacción & $.326^{*}$ & $.561^{*}$ & -.043 \\
Amor & $.415^{\star}$ & $.310^{*}$ & .244 \\
Tristeza & $.479^{*}$ & .244 & $.357^{\star}$ \\
Ira & $.690^{*}$ & $.356^{*}$ & $.509^{*}$ \\
Miedo & $.558^{*}$ & .002 & $.645^{\star}$ \\
Empatía & .141 & .162 & .036 \\
Culpa & $.360^{*}$ & -.078 & $.459^{\star}$ \\
Vergüenza & $.465^{*}$ & -.160 & $.645^{\star}$ \\
Correlación & & & \\
F1 & - & & \\
F2 & - & $.80^{*}$ & \\
\hline
\end{tabular}

${ }^{*} p<.05$ 
Respecto a la Dimensionalidad del instrumento, se examinó la validez de la estructura interna y de contenido del instrumento. Estos dos aspectos de la validez son elementales y condicionantes de evidencias de validez posteriores, dado que estructuran la calificación de los puntajes y la correspondencia entre la estructura hipotetizada y la estructura empírica. De acuerdo a la construcción del instrumento, su estructura hipotetizada consistió en tres constructos generales, que contenían emociones específicas: emociones positivas (alegría, interés, satisfacción y amor), emociones negativas (tristeza, ira y miedo), y emociones morales (empatía, culpa y vergüenza). Dado que cada emoción específica contenía dos ítems de calificación dicotómica $\left(\begin{array}{lll}1 & \circ & 0\end{array}\right)$, el rango de puntuación fue de 0 a 2 en cada emoción. Esto estableció que la variable observada era ordinal con reducido número rango de respuesta, lo que indicaba que una metodología de variables categóricas era adecuada para explorar la dimensionalidad (Lei, 2009; Raykov, 2012). Para ello se usó el estimador weighted least squares means and variance adjusted (WLSMV; Muthén, 1984; Muthén, du Toit, y Spisic, 1997) con correlaciones policóricas, que tienden a ser apropiados cuando el rango de opciones es restringido, esto es, menor a cinco opciones. El método analítico fue el modelamiento de ecuaciones estructurales exploratorio (ESEM), con rotación Geomin (Asparouhov, y Muthén, 2009); para el ajuste se obtuvo la prueba estadística de tipo $\square 2$ (WLSMV- $\square 2$ ) e indicadores de ajuste prácticos, como CFI ( $\geq$.95), RMSEA ( $\leq .05 ;$ IC 90\%). Se usó el programa MPLUS 7 (Muthén, y Muthén, 1998-2012) para este análisis.

La consistencia $(\alpha)$ fue de .814 para la sub escala de emociones positivas, .773 para la emociones negativa y .836 para la sub escala de emociones morales. De la misma manera, la confiabilidad del instrumento se obtuvo también mediante las respuestas de los ítems en el grupo control en el pre y post-evaluación, las que se consideraron como evidencia de estabilidad de los puntajes (confiabilidad test re-test). La estabilidad de los puntajes como estimación de la confiabilidad es perfectamente relevante a la situación experimental aplicada, pues se consideró que las respuestas del grupo control fueron fundamentalmente registros de los niños/as en una situación social natural y no intervenida sistemáticamente por la aplicación del programa u otro factor explícito de cambio. El periodo de tiempo entre las respuestas en la pre y post evaluación en el grupo control fue igual que para el grupo experimental, así que la estabilidad de los puntajes tiene un marco de interpretación vinculado con el programa.

\section{Procedimiento}

Inicialmente se realizaron las coordinaciones con las Directores de los centros educativos y las docentes de aula para que autorizaran la participación. La selección de los centros educativos en los que se desarrolló el programa se realizó de acuerdo con la disponibilidad de los directivos y del profesorado. Se programó una reunión con los padres/madres de familia, quienes luego de un taller, llenaron el Consentimiento Informado en donde se daba a conocer el propósito de la investigación, así como la aprobación para que el niño(a) participe en el estudio.

La implementación del programa se realizó en el mismo salón de clases y las sesiones fueron ejecutadas por un grupo de cinco estudiantes de psicología de los últimos ciclos, debidamente capacitadas para este fin. Inicialmente el equipo de estudiantes realizó una estadía en el campo por aproximadamente tres visitas previas, en donde interactuaron con los niños/as durante las horas de clases y el recreo. El programa fue supervisado por la autora del estudio. La aceptabilidad de la aplicación del instrumento por parte de los niños/as del grupo experimental y control fue favorable.

En cuanto al Procedimiento experimental, el programa se desarrolló en diez sesiones, con una duración de dos horas académicas, cuarenta y cinco minutos cada una, organizadas en tres módulos, mediante la aplicación de técnicas participativas y lúdicas. Se desarrolló una vez por semana, con una duración de diez semanas en total y el objetivo general del programa fue 
reconocer y expresar las emociones positivas y morales, y el manejo de emociones negativas. Los módulos que se desarrollaron fueron los siguientes: Módulo 1: Mis emociones positivas (sesión 1: Alegría; sesión 2: Interés; sesión 3: Satisfacción y sesión 4: Amor). La alegría, constituye una intensa emoción caracterizada por diversión y regocijo (Lazarus, 2000), que ayuda a construir recursos individuales que perdurarán mucho después de que la conducta haya terminado (Fredrickson y Branigan, 2000). El interés, considerado como la exploración explícita y activa de aumentar conocimientos y experiencias que se dan mediante la incorporación de nueva información (Ccaypani, 2011) y se asocia con el fortalecimiento de la motivación para completar las tareas que son tediosas (Paul, 2006). La satisfacción, que corresponde el bajo nivel de la euforia, la plenitud o el triunfo (Fernández-Abascal, 2016), permite el disfrute, lo que se traduce en un nivel óptimo de autoconcepto (Fredrickson, 1998) y el amor. Al respecto, las experiencias de amor están compuestas de emociones positivas y ayudan a fortalecer la cohesión social y el apego.

En el Módulo 2 se consideró a las emociones negativas (sesión 5: Tristeza; sesión 6: Ira y sesión 7: Miedo). La tristeza: se asocia con la pérdida de esperanza, la desmotivación para actuar y el dolor moral, entre otras dificultades. La ira, considerada como un estado de malestar que puede ir desde una intensidad equivalente a la irritación suave, hasta la denominada cólera intensa (Izzedin y Cuervo, 2009), y puede surgir como una reacción a la vulnerabilidad ante una amenaza o daño recibido, ya sean reales o imaginarios (Moral, Gonzáles y Landero, 2011). El miedo, se activa por la percepción de daño o peligro (Piqueras, et al., 2009).

En el Módulo 3, se abordaron las emociones morales (sesión 8: Empatía; sesión 9: Culpa y sesión 10: Vergüenza). La empatía, constituye una respuesta afectiva que hace posible la comprensión de la condición o estado emocional de otra persona. Incluye, tanto respuestas emocionales como la capacidad para entender los estados afectivos de los demás (Eisenberg, 2000; Garaigordobil y García, 2006). La culpa, se caracteriza por evaluaciones y el sentir que se genera cuando hay un mal comportamiento (Turan y Cohen, 2016), implica una sensación de arrepentimiento y motiva la realización de acciones reparadoras. (Cavalera y Pepe, 2014). La vergüenza, se caracteriza por las autoevaluaciones negativas, es decir sentir que uno es una persona imperfecta y con pérdida de dignidad (Tracy y Robins, 2006).

Las técnicas que se utilizaron fueron diversas y con diferentes objetivos durante el desarrollo de la sesión, tales como: Introducción y motivación (lluvia de ideas, audiciones musicales y técnicas participativas), desarrollo (técnicas participativas de actuación, técnicas de relajación, ejercicios de aplicación y videos), evaluación (ejercicios aplicativos), monitoreo (formato breve) y despedida (técnicas participativas).

En todas las sesiones se inició con una actividad motivadora, luego se desarrollaron los contenidos, y finalmente la evaluación y monitoreo, el cual se realizó para reorientar las actividades si fueran necesarios.

Es necesario señalar que se respetaron los principios éticos del capítulo III, título Investigación del Código de Ética del Perú (Colegio del Psicólogo del Perú, 2017) referidos al respeto de la normatividad internacional y nacional que regula la investigación en humanos, confidencialidad, principio de libertad, entre otros.

\section{Análisis de datos}

Se hicieron dos análisis, respecto a las diferencias de los grupos en el pretest, y el efecto de la intervención.

En cuanto a las diferencias en el pretest, debido que los grupos de tratamiento provinieron de 
dos instituciones distintas, se hizo un análisis de los puntajes en el pretest de éstos, incluyendo el grupo control. El análisis de hizo con la prueba $t$ Student para grupos dependientes, y el tamaño del efecto se basó en lo propuesto por Plonsky y Oswald (2014) para comparaciones intragrupo (.60 para pequeño, 1.0 para moderado, y 1.4 para grande) y entre-grupo (.40 para pequeño, .70 para moderado, y 1.0 para grande).

Efectos de la intervención. El análisis se hizo en dos enfoques: el de efectos grupales, y de efectos individuales. Para ver el efecto de grupo, se utilizó un ANOVA dos-vías de medidas repetidas (suma de cuadrados Tipo III), con el tipo de institución (tres instituciones: IN, VI y CAS) como factor entre-sujetos y el puntaje en el instrumento (dos puntajes: pretest y postest) como factor intra-sujetos. El tamaño del efecto fue estimado con eta cuadrado parcial generalizado para medidas repetidas (Bakeman, 2005), y eta cuadrado parcial (Lakens, 2013) para el contraste entre-grupos. Para ambos, se utilizó el programa MOTE (Buchanan, Padfield, Van Nuland, Wikowsky, y Gillenwaters, 2018). El cambio en la distribución de los puntajes también fue informado mediante el coeficiente de solapamiento BC (Bhattacharyya, 1943; Brown, 2015).

Para la evaluación de los efectos individuales del tratamiento, se adoptaron dos estrategias. Primero, el reporte descriptivo del cambio individual fue apoyado por el porcentaje de cambio de mejora $(\mathrm{PdC})$ calculado como la división entre la diferencia entre el puntaje del postest y del pretest, relativo al puntaje pre-test, y este resultado multiplicado por 100 (Hiller, Schindler y Lambert, 2012). En el presente estudio, valores negativos indicaron que el cambio ocurrió hacia debajo del puntaje del pre-test, indicando deterioro. Segundo, el efecto de la intervención se analizó mediante la significancia del cambio individual, con el método de Jacobson y Truax (JT, 1991), en que se toman dos aspectos definitorios del CCS, el cambio confiable y el cambio clínico significativo (CCS). El cambio confiable fue estimado mediante el índice de cambio confiable (RCl; Jacobson y Truax, 1991). Debido la ausencia de normas para clínicas o para una muestra numerosa o representativa en el instrumento utilizado, se aplicó el criterio del enfoque JT, en que el puntaje en el postest debería ser dos desviaciones estándares del puntaje pretest (1.96 en el $95 \%$ de confianza). Sin embargo, el criterio puede ser muy restrictivo dado la confiabilidad del instrumento, el contenido muestreado por el instrumento, la investigación emergente del tratamiento implementado y el incremento de falsos negativos. Una adecuación de este criterio puede ser recomendable cuando se evalúan estos aspectos (Wise, 2004), y por lo tanto el criterio de cambio fue establecido en una desviación estándar del pretest multiplicado por 1.28 (90\% de confianza), específicamente $\mathrm{M}$ (media) + 1.28*DE. Este análisis se aplicó por separado, y entonces la $M$ y la $D E$ se obtuvieron de cada grupo experimental (VI e IN) y control (CAS).

En conjunto, el $\mathrm{PdC}$ y $\mathrm{RCl}$ pueden ser informaciones efectivas para evaluar el efecto individual del tratamiento (Chomycz y Schmidt, 2015), y fueron evaluados respecto a su convergencia (Middel, Stewart, Bouma, van Sonderen y van den Heuvel, 2001; Middel y van Sonderen, 2002). Para estimar los indicadores clinicométricos, se utilizó la correlacion test-retest proveniente del grupo control, dado que es recomendada esta muestra para obtener la estabilidad de las medidas (Howard, Lueger, Maling, y Martinovich, 1993).

\section{Resultados}

En cuanto a las diferencias entre el grupo de intervención (en conjunto, IN y $\mathrm{VI}$ ) y el grupo control (CAS) fue alrededor de cero (diferencia $=.377$, IC $95 \%=-.621,1.375$ ), estadísticamente no significativa $t=.751$, Satterthwaite-gl $=80.77)$ y trivial $(g=.122, I C 95 \%=-.286, .530)$. Efectivamente, el solapamiento de distribuciones entre los puntajes del pretest fue alta $(B C=$ 
.916). Sin embargo, el análisis de medias de los tres grupos arrojó diferencias más allá del error de muestreo, Welch- $F(2,43.74)=3.729, p<.05$, y de tamaño pequeño, $f=.087$.

En una comparación post-hoc, se detectó que en uno de los grupos de intervención (IN) difirió moderadamente respecto el otro grupo de intervención $(g=.755, \mathrm{IC} 95 \%=.209,1.288 ; t=2.698$, $\mathrm{gl}=24.95, p<.05$ ), y del grupo control CAS (diferencia grande, $g=.833$, IC 95\% $=.217,1.434 ; t=$ $2.559, \mathrm{gl}=25.31, p<.05)$. Por otro lado, el grupo de intervención VI y el CAS fueron similares en sus medidas, (diferencia trivial, $g=-.035$, IC 95\% $=-.454, .384 ; t=-.210, \mathrm{gl}=80.01, p>.10$ ). Dada estas diferencias parciales en el pre-test, ambos grupos de intervención en el pretest fueron tratados independientemente.

Tabla 1

Resultados descriptivos de los puntajes en el pretest y postest

\begin{tabular}{|c|c|c|c|c|c|c|c|c|c|c|}
\hline & \multirow[b]{2}{*}{$\mathbf{M}$} & \multicolumn{4}{|c|}{ Puntaje pretest } & \multicolumn{5}{|c|}{ Puntaje postest } \\
\hline & & $+\mathrm{N}$ & DE & As. & $\mathrm{Cu}$ & M & & DE & As. & $\mathrm{Cu}$ \\
\hline \multicolumn{11}{|l|}{$\begin{array}{l}\text { Grupos de } \\
\text { intervención }\end{array}$} \\
\hline $\begin{array}{l}\text { IN }(n=19) \\
\mathrm{VI}(n=76)\end{array}$ & $\begin{array}{l}6.95 \\
4.53\end{array}$ & $\begin{array}{l}19 \\
76\end{array}$ & $\begin{array}{l}3.597 \\
3.070\end{array}$ & $\begin{array}{r}.056 \\
1.058\end{array}$ & $\begin{array}{r}-1.422 \\
1.164\end{array}$ & $\begin{array}{l}18.37 \\
17.95\end{array}$ & $\begin{array}{l}9 \\
6\end{array}$ & $\begin{array}{l}1.674 \\
2.274\end{array}$ & $\begin{array}{r}-.896 \\
-1.226\end{array}$ & $\begin{array}{r}-.217 \\
1.092\end{array}$ \\
\hline \multicolumn{11}{|c|}{ Grupo Control } \\
\hline $\operatorname{CAS}(n=30)$ & 4.63 & 30 & 2.025 & .540 & -.160 & 6.33 & 0 & 2.023 & .367 & -.406 \\
\hline
\end{tabular}

Nota. As.: coeficiente de asimetría. Cu.: coeficiente de curtosis.

\section{Figura 1}

Cambio en el puntaje promedio, del pretest al postest

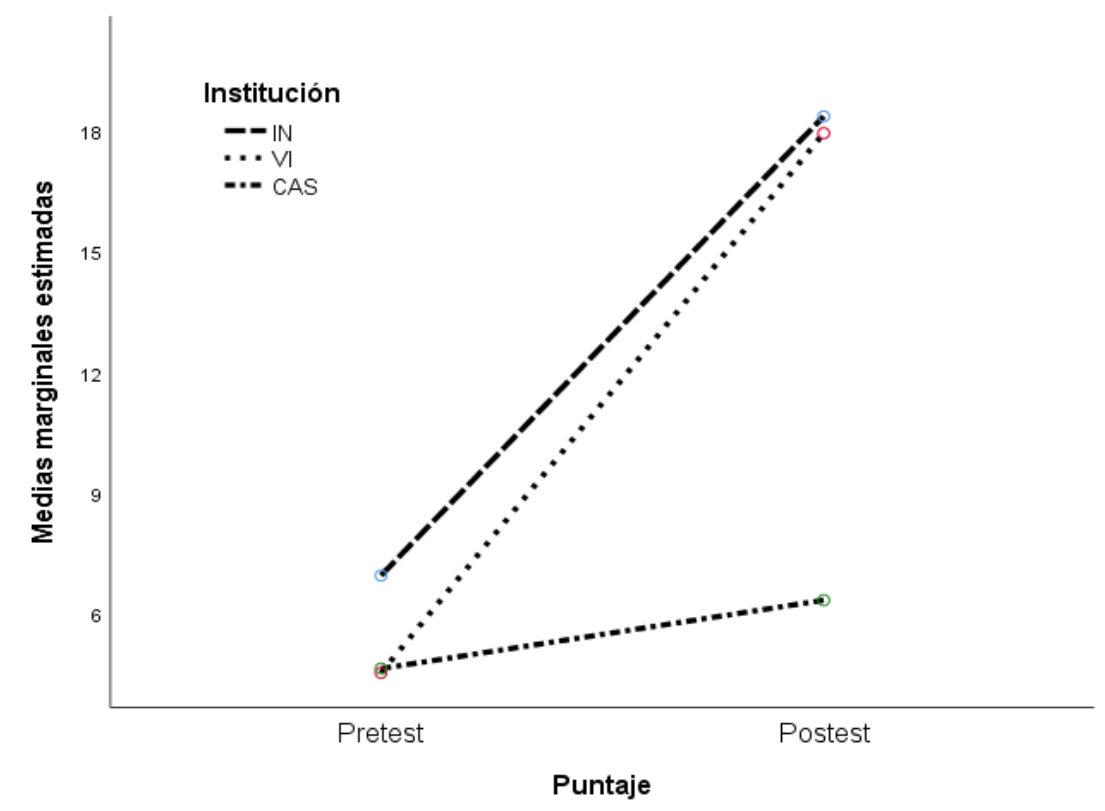

Con respecto a los efectos en el tratamiento, las diferencias de grupo, el análisis reveló que el efecto principal entre-grupos de la institución educativa fue estadísticamente significativo, 
$\mathrm{F}(2,122)=111.975, \mathrm{p}<.01$, y de magnitud grande $\left(\eta_{p}^{2}=.65\right.$, IC 95\% $\left.=.53, .73\right)$. El efecto principal intragrupo de la medidas pretest-postest también fue estadísticamente significativo, $\mathrm{F}(1$, $122)=640.637, \mathrm{p}<.01$, y fue un efecto grande $\left(\eta_{p G}^{2}=.69, \mathrm{IC} 95 \%=.58, .77\right)$. Y el efecto de interacción fue estadísticamente significativo, $F(2,122)=134.443(p<.01)$, también de gran magnitud $\left(\eta_{p G}^{2}=.48\right.$, IC $\left.95 \%=.34, .59\right)$. Esto indicó que la condición de tratamiento tuvo un efecto moderador en los puntajes intragrupos, del pretest al postest. Esto se muestra mejor en la Figura 1, donde los grupos de intervención (IN y VI) mostraron un elevado puntaje postest promedio, mientras que el control (CAS) mostró también un puntaje promedio mayor en el postest, pero fue menor. Los puntajes promedio del postest en los grupos convergieron en magnitud y cerca del máximo puntaje posible. También se observó que la variabilidad de los grupos de intervención fue menor en la evaluación pretest, comparado con la evaluación postest.

En cuanto a las diferencias individuales, se estimaron el error estándar de medición, el puntaje pretest mínimo para establecer el cambio significativo clínico (criterio a ajustado en el $90 \%$ de confianza, $z=1.28)$, y el $\mathrm{RCl}$ para cada institución.

Usando el criterio ajustado para la institución IN, el error estándar de medición fue 2.20, el criterio de cambio clínico fue 11.55 , con un $\mathrm{RCI}=6.09$; para la institución $\mathrm{VI}$, el error estándar de medición fue 1.88; el criterio de cambio clínico fue 8.45 , con un $\mathrm{RCl}=5.20$. Para el grupo control (CAS), el criterio fue 7.22 , con un $\mathrm{RCl}=3.43$. En la Tabla 3 se observan estas diferencias.

\section{Tabla 3}

Resultados clinicométricos: Cambio confiable y clínico

\begin{tabular}{|c|c|c|c|c|}
\hline & \multicolumn{2}{|c|}{$\begin{array}{c}\text { Cambio confiable } \\
\text { (distribución en RCl) }\end{array}$} & \multicolumn{2}{|c|}{$\begin{array}{c}\text { Cambio clínico } \\
\text { (criterio a ajustado) }\end{array}$} \\
\hline & $N$ & $\%$ & $\begin{array}{l}\text { Porcentaje de } \\
\text { mejora (cambio) }\end{array}$ & $\operatorname{ccs}$ \\
\hline \multicolumn{5}{|c|}{ Grupo de intervención (experimental) } \\
\hline \multicolumn{5}{|c|}{$\mathrm{IN}(n=19)$} \\
\hline Mejora & 18 & 94.7 & $\begin{array}{l}\operatorname{Min}=58.3 \% \\
\operatorname{Max}=850.0 \%\end{array}$ & $100.0 \%$ \\
\hline No cambio & 1 & 5.2 & $\begin{array}{l}\operatorname{Min}=41.7 \% \\
\operatorname{Max}=58.2 \%\end{array}$ & $0.0 \%$ \\
\hline Deterioro & 0 & 0.0 & $41.7 \%<$ & $0.0 \%$ \\
\hline \multicolumn{5}{|l|}{$\mathrm{VI}(n=76)$} \\
\hline Mejora & 74 & 97.3 & $\begin{array}{l}\operatorname{Min}=54.5 \% \\
\operatorname{Max}=900 \%\end{array}$ & $100.0 \%$ \\
\hline No cambio & 2 & 2.6 & $\begin{array}{l}\operatorname{Min}=26.7 \% \\
\operatorname{Max}=50.0 \%\end{array}$ & $0.0 \%$ \\
\hline Deterioro & 0 & 0.0 & $26.0 \%<$ & $0.0 \%$ \\
\hline \multicolumn{5}{|l|}{ Grupo control } \\
\hline $\begin{array}{l}\text { CAS }(n=30) \\
\text { Mejora }\end{array}$ & 3 & 10.0 & $\begin{array}{l}\text { Min }=83.3 \% \\
\operatorname{Max}=166.7 \%\end{array}$ & $3.3 \%$ \\
\hline No cambio & 27 & 90.0 & $\begin{array}{l}\operatorname{Min}=-25.0 \% \\
\operatorname{Max}=300.0 \%\end{array}$ & $96.6 \%$ \\
\hline Deterioro & 0 & 0.0 & $-25.0 \%<$ & $0.0 \%$ \\
\hline
\end{tabular}

Nota. RCl: índice de cambio confiable. CCS: cambio clínico significativo según criterio a ajustado en el $90 \%$ de confianza $(z=1.28)$. 


\section{Discusión}

El presente programa ha demostrado generar cambios en los niños/as evidenciado a partir de los puntajes obtenidos al final de la intervención. En la evaluación inicial, no se encontraron diferencias significativas entre el grupo control y experimental, pues ambos grupos obtuvieron puntajes homogéneos y bajos en relación con el reconocimiento y expresión de emociones positivas, negativas y morales.

Los resultados muestran que los niños/as del grupo experimental, tras la realización del programa, reconocen con mayor exactitud las emociones que fueron trabajadas. Estos resultados se relacionan con los programas aplicados en niños/as con edad preescolar en habilidades relacionadas a la empatía y el manejo de emociones (Ambrona, López-Pérez y MárquezGonzález; 2012; Amesty y Clinton, 2009; Celdrán y Ferrándiz, 2012; McMahon, Washburn, Felix, Yakin y Childrey, 2000; Obiols, 2005).

En el caso del módulo de emociones positivas, los niños/as lograron reconocerlas y expresarlas, lo cual resulta útil para aumentar la resiliencia ante eventos estresantes (Tugade y Fredrickson, 2007; Fredrickson, 2013). Además estas emociones se producen y perciben durante la infancia (Hoehl y Striano, 2010) y su estimulación, mediante el lenguaje y la influencia del grupo, influyen en este proceso de nombrarlas (Lindquist, MacCormack y Shablack, 2015). De esta manera, las estrategias orientadas a la participación podrían haber influido en los resultados.

Con respecto a las emociones morales, podría sorprender la intervención en una población infantil como la del presente estudio; sin embargo diversos estudios han demostrado también que los niños/as en edad preescolar presentan conductas prosociales, tal como se ha demostrado en otros estudios (Cooley, Elenbaas y Killen, 2012; Garaigordobil, 2003; Seco, 2006). Constituyen moderadores potenciales del vínculo ira-agresión (Colasante, Zuffianò y Malti, 2015) y ayudan a los niños/as a anticipar las consecuencias negativas de su comportamiento (Arsenio, 2014).

Un aspecto importante ha sido la continuidad del programa; es decir se cumplió con el protocolo científico con el que fue elaborado, tal como lo sugieren Pichardo, García, Justicia y Llanos (2008). El cumplimiento del procedimiento y cronograma, al parecer resultaron importantes en este proceso; así como la recuperación de alguna de las sesiones en el caso de algún niño que faltó hasta una sesión. Otro aspecto constituye las técnicas utilizadas, en este caso el uso de técnicas participativas ha favorecido el aprendizaje (Levy yThompson, 2013; Clarke, Sixsmith y Barry, 2014; Martín, 2002), éstas involucran actividades lúdicas, movimientos corporales, juegos, dinámicas, en las cuales los niños/as participan de forma activa (Campoverde y Naranjo, 2012). Estas técnicas son utilizadas para poder expresar emociones, sentimientos y temores (García, 2003), y facilitan la integración de los miembros de un grupo (Montañés, 2009).

Como aspecto positivo, se percibió una acogida del programa por parte de los directores, docentes y auxiliares de educación, al señalar que las técnicas y estrategias utilizadas fueron las apropiadas para la edad de los niños/as/niñas, y en muchos casos novedosas, como es el uso de las técnicas participativas orientadas a la dinamización (Montañés, 2011). En nuestro medio, el Ministerio de Educación (2019) señala que "los docentes de Educación Inicial deben promover que las niñas y niños/as establezcan vínculos emocionales estables" (p.14), la capacitación en estos temas es escaza, lo cual impacta en la formación docente, la misma que requiere ser permanente (Didonet, 2007). Otro aspecto positivo a destacar, constituye la asistencia regular en 
la mayoría de los participantes, a quienes se les observaron motivados para participar en cada una de las sesiones. De la misma manera, la valoración positiva por parte de los padres/madres de familia al llevar puntualmente a sus niños/as para que participen en el programa.

\section{Conclusiones $^{2}$}

Por todo lo anteriormente señalado, se concluye que los niños/as que participaron en el programa lograron reconocer y expresar las emociones positivas y morales, y manejar las emociones negativas. Así se demuestra la efectividad del programa y se comprueba la hipótesis formulada.

Resulta evidente la necesidad de ejecutar programas destinados al desarrollo y mejora del mundo emocional, pues su importancia debe ser contemplada como un contenido más a educar para favorecer el desarrollo integral de las personas (Colasante, et al. 2015; López, 2005). Intervenir a edades tempranas posibilita el desarrollo de conductas saludables en el futuro (Garaigordobil, 2003). En cuanto a las limitaciones del presente estudio, constituye el tipo de muestreo, en la medida que no pueden generalizarse los resultados. De la misma manera, el uso de un solo instrumento, quizás hubiera sido necesario incluir una escala pictórica o un instrumento similar.

Para futuras investigaciones se sugiere implementar programas en otros grupos muestrales; por ejemplo, en grupos que asisten a terapia o grupo de niños/as institucionalizados y en muestras más amplias. Así mismo, utilizar estrategias para realizar un seguimiento a los participantes con la finalidad de establecer la estabilidad en el tiempo de las habilidades emocionales adquiridas. En esta misma línea, entrenar a los docentes para que actúen como facilitadores del programa, pues la intervención temprana en el manejo de las emociones resulta un factor protector para el caso de los niños/as. Una propuesta constituye involucrar a los padres y madres de familia, pues de esta manera los resultados del programa se potencializan y faciliten la transferencia del aprendizaje. Se sugiere abordar la sostenibilidad de la implementación de un proyecto de educación emocional en las escuelas participantes. Desde una perspectiva más amplia, se sugiere generar una política en los centros atendidos, de tal manera que tenga un mayor impacto en el desarrollo socio afectivo del educando, considerando el desarrollo de una educación integral y orientada a la convivencia social.

\section{Referencias bibliográficas}

Adler, A. (2017). Educación Positiva: Educando para el éxito académico y para la vida plena. Papeles del psicólogo, 38(1), 50-57, doi: 10.23923/pap.psicol2017.2821

\footnotetext{
${ }^{2}$ Los autores quieren mostrar su agradecimiento a las docentes de los centros educativos que conformaron la muestra por su apoyo en el desarrollo del programa.
} 
Alarcón, R. (2008). Métodos y Diseños de Investigación del Comportamiento. Lima: Universidad Ricardo Palma.

Ambrona, T., López-Pérez, B. y Márquez-González, M. (2012). Eficacia de un programa de Educación emocional breve para incrementar la competencia emocional de niños/as en educación primaria, Revista Española de Orientación y Psicopedagogía, 23(1), 39-49. doi:10.5944/reop.vol.23.

Amesty, E. y Clinton, A. (2009). Adaptación Cultural de un programa de Prevención a nivel preescolar. Interamerican Journal of Psychology, 43(1), 106-103.

Arsenio, W. (2014). Moral emotion attributions and aggression. In M. Killen, y J. Smetana (Eds.), Handbook of moral development (2nd ed., pp. 235-255). New York: Psychology Press.

Asparouhov, T. y Muthén, B. (2009). Exploratory structural equation modeling. Structural Equation Modeling, 16, 397-438, doi:10.1080/10705510903008204

Atkins, D. C., Bedics, J. D., McGlinchey, J. B., y Beauchaine, T. P. (2005). Assessing clinical significance: Does it matter which method we use? Journal of Consulting and Clinical Psychology, 73, 982-989, doi:10.1037/0022-006X.73.5.982.

Ato E., González C., y Carranza J.A. (2004). Aspectos evolutivos de la autorregulación emocional en la infancia. Revista Anales de Psicología, 20(1), 69-79.

Bakeman, R. (2005). Recommended effect size statistics for repeated measures designs. Behavior Research Methods, 37, 379-384, doi: 10.3758/BF03192707

Bauer, S., Lambert, M. J., y Nielsen, S. L. (2004). Clinical significance methods: A comparison of statistical techniques. Journal of Personality Assessment, 82(1), 60-70, doi:10.1207/s15327752jpa8201_11

Beurs, E., Barendregt, M., Heer, A., Duijn, E., Goeree, B., Kloos, M., Kooiman, K., Lionarons, H., y Merks, A., (2015). Comparing methods to denote treatment outcome in clinical research and benchmarking mental health care. Clinical Psychology y Psychotherapy, 23(4), 308-318, doi:10.1002/cpp.1954

Bhattacharyya, A. (1943). On a measure of divergence between two statistical populations defined by their probability distributions. Bulletin of the Calcutta Mathematical Society, 35, 99-109.

Bisquerra, R. (2005). La Educación emocional en la formación del profesorado. Revista Interuniversitaria de formación del profesorado, 19(13), 95-114.

Brown, G. K. (2015). BHATT: Stata module to compute Bhattacharyya Coefficient and Bhattacharyya Distance statistics of distribution overlap. Recuperado de https://econpapers.repec.org/software/bocbocode/s458091.htm (5 agosto 2018)

Bruce, V., y Young, A. (1986). Understanding face recognition. British journal of Psychology, 77(3), 305-327, doi:10.1111/j.2044-8295.1986.tb02199.x

Buchanan, E. M., Padfield, W. E., Van Nuland, A., Wikowsky, A., y Gillenwaters, A. (2018). MOTE: The Shiny App to Calculate Effect Sizes and Their Confidence Intervals. Recuperado de: https://osf.io/tds83/

Campoverde, A.P. y Naranjo, B.M. (2012). Manual de Técnicas Participativas en el trabajo de educadores populares para fomentar la creatividad en los niños/as/as de 7 y 8 años de la Fundación DS y $\mathrm{H}$. (Tesis para optar del título de psicólogo). Universidad Politécnica Salesiana, Quito, Ecuador. 
Catalino L. y Fredrickson, B. (2011). A Tuesday in the Life of a Flourisher: The Role of Positive Emotional Reactivity in Optimal Mental Health. Emotion 11(4), 938-950, doi:10.1037/a0024889

Cavalera, C. y Pepe, A. (2014). Social emotions and cognition: shame, guilt and working memory. Procedia. Social and Behavioral Sciences, 112, 457-464, doi:10.1016/j.sbspro.2014.01.1189

Ccaypani, L. (2011). Conservación de un jardín y generación de emociones positivas en preadolescentes de San Juan de Miraflores. (Tesis para optar el Título de Licenciada en Psicología). Universidad de San Martín de Porres, Lima, Perú.

Celdrán, J. y Ferrándiz, C. (2012). Reconocimiento de emociones en niños/as de Educación Primaria: Eficacia de un programa educativo para reconocer emociones. Electronic Journal of Research in Educational Psychology, 10(28), 1321-1342.

Chóliz, M. (2005). Psicología de la emoción: el proceso emocional. Recuperado de www.uv.es/=choliz

Chomycz, S., y Schmidt, F. (2015). Practice guidelines for the assessment of clinically significant treatment outcomes in the Children's Mental Health System. Journal of Evidence-Informed Social Work, 13(2):236-248, doi:10.1080/23761407.2015.1031417

Colasante, T., Zuffianò, A., y Malti, T. (2015). Do moral emotions buffer the anger-aggression link in children and adolescents? Journal of Applied Developmental Psychology, 41, 1-7. doi:10.1016/j.appdev.2015.06.001

Colegio del Psicólogo del Perú (2017). Código de Ética y Deontología. Lima: Autor.

Cooley, S., Elenbaas, L., y Killen, M. (2012). Moral judgments and emotions: Adolescents' evaluations in intergroup social exclusion contexts. New Directions for Youth Development, 136, 41-57, doi:10.1002/yd.20037.

Denham, S. A., Bassett, H. H., Brown, C., Way, E., y Steed, J. (2013). "I know how you feel": Preschoolers' emotion knowledge contributes to early school success. Journal of Early Childhood Research, 221-235. Advance online publication, doi: 10.1177/1476718X13497354

Didonet, V. (2007). Formación de profesores para Educación Inicial. Revista de Investigación, 31(62), 15-40.

Eisenberg, N. (2000). Emotion, Regulation and Moral Development. Annual Review of Psychology, 51, 665-697. doi:10.1146/annurev.psych.51.1.665

Escurra, L.M. (1988). Cuantificación de la validez de contenido por criterio de jueces. Revista de Psicología, 6(1-2), 103-111.

Fernández-Abascal, E. (2016). Disfrutar de las emociones positivas: Psicología. Madrid: Editorial Grupo 5.

Fredrickson, B. L. (1998). What good are positive emotions? Review of General Psychology, 2 (3), 300-319, doi:10.1037/1089-2680.2.3.300

Fredrickson, B. L. (2001). The role of positive emotion in positive psychology: The broaden and build theory of positive emotion. American Psychologist, 56, 218-226, doi:10.1037/0003066X.56.3.218

Fredrickson, B. L. (2013). Positive emotions broaden and build. In P. Devine y A. Plant (Eds.), Advances in experimental social psychology (Vol. 47, pp. 1-54). San Diego: Academic Press. 
Fredrickson, B. L. (2018). Biological Underpinnings of Positive Emotions and Pusrpose. In Forgas, J. y Baumeister, R.F. (Eds), The Social Psychology of Living Well. The Sydney Symposium of Social Psychology (pp.163-180), New York: Taylor y Francis

Fredrickson, B. L. y Branigan, C. (2000). Positive emotions. En T. J. Mayne y G. A. Bonanno (Eds.), Emotion: current issues and future directions (pp. 123-151). Nueva York: Guilford Press.

Fredrickson, B. L. y Joiner, T. (2002). Positive emotions trigger upward spirals toward emotional wellbeing. Psychological Science, 12(2), 172-175, doi:10.1111/14679280.00431

Fredrickson, B. L.; Mancuso, R. Branigam, C y Tugade, M. (2000). The undoing effect of positive emotions. Motivation and Emotion, 24(4), 237-258, doi: 10.1023/A: 1010796329158

Garaigordobil, M. (2003). Intervención psicológica para desarrollar la personalidad infantil: Juego, conducta prosocial y creatividad. Madrid: Pirámide.

Garaigordobil, M., y García, P. (2006). Empatía en niños/as de 10 a 12 años. Psicothema, 18, 180186.

García, D. (2003). El grupo métodos y técnicas participativas. Buenos Aires: Espacio Editorial.

Haidt, J. (2007). The new synthesis in moral psychology. Science, 316, 998-1002, doi:10.1126/science.1137651

Hafkenscheid, A. (2000). Psychometric measures of individual change: An empirical comparison with the Brief Psychiatric Rating Scale (BPRS). Acta Psychiatrica Scandinavia, 101, 235-242, doi:10.1046/j.0902-4441.2000.ap90076.x

Havighurst, S. S., Wilson, K. R., Harley, A. E. y Prior, M. R. (2009). Tuning in to kids: an emotionfocused parenting program-initial findings from a community trial. Journal of Community Psychology, 37(8), 1008-1023, doi:10.1002/jcop.20345

Haxby, J. V., Hoffman, E. A., y Gobbini, M. I. (2000). The distributed human neural system for face perception. Trends in cognitive sciences, 4(6), 223-233.

Heinze, J. E., Miller, A. L., Seifer, R., Dickstein, S. y Locke, R. L. (2015). Emotion knowledge, loneliness, negative social experiences, and internalizing symptoms among low income preschoolers. Social Development, 24, 240-265, doi:10.1111/sode.12083

Hilt L.M., Hanson J.L., y Pollak S.D. (2011). Emotion Dysregulation. Encyclopedia of Adolescence, 3, 160-169.

Hicks, J.A., Schlegel, R.J. y King, L.A. (2010). Social threats, happiness, and the dynamics of meaning in life judgments. Personality and Social psychology Bulletin, 36(10), 1305-1317, doi:10.1177/0146167210381650

Hiller, W., Schindler, A. C., y Lambert, M. J. (2012). Defining response and remission in psychotherapy research: A comparison of the $\mathrm{RCl}$ and the method of percent improvement. Psychotherapy Research, 22, 1-11, doi:10.1080/10503307.2011.616237

Hoehl, S., y Striano, T. (2010). The development of emotional face and eye gaze processing. Developmental Science, 13, 813-825, doi:10.1111/j.1467-7687.2009.00944.x.

Hoffman, E. A., y Haxby, J. V. (2000). Distinct representations of eye gaze and identity in the distributed human neural system for face perception. Nature neuroscience, 3(1), 80-84, doi: $10.1038 / 71152$ 
Howard, K. I., Lueger, R. J., Maling, M. S., y Martinovich, Z. (1993). A phase model of psychotherapy outcome: Causal mediation of change. Journal of Consulting and Clinical Psychology, 61(4), 678-685, doi:10.1037/0022-006X.61.4.678

Izzedin, R. y Cuervo, A. (2009). Conceptualización, factores asociados y estrategias de autorregulación de la tristeza en niños/as y niñas de 10 años. Tesis Psicológica, 4, 52-71.

Jacobson, N. S., y Truax, P. (1991). Clinical significance: A statistical approach to defining meaningful change in psychotherapy research. Journal of Consulting and Clinical Psychology, 59, 12-19.

Kohlberg, L. (1984). Essays on moral development. San Francisco: Harper y Row.

Lauw, M., Havighurst, S., Wilson, K., Harley, A. y Northam, E. (2014). Improving parenting of toddler's emotions usinf an emotion coaching parenting program: a pilot study of tuning in to toddlers. Journal of Community Psychology, 42(2), 169-175. doi:10.1002/jcop.21602

Lakens, D. (2013). Calculating and reporting effect sizes to facilitate cumulative science: a practical primer for t-tests and ANOVAs. Frontiers in psychology, 4, 863, doi:10.3389/fpsyg.2013.00863.

Lazarus, R.S. (2000). Estrés y emoción. Manejo e implicancias en nuestra salud. Barcelona: Desclée de Brouwer.

Lei, P. (2009). Evaluating estimation methods for ordinal data in structural equation modeling. Quality and Quantity, 43, 495-507, doi:10.1007/s11135-007-9133-z

Levy, R., y Thompson, P. (2013). Creating "buddy partnerships" with 5- and 11-year old-boys: a methodological approach to conducting participatory research with young children. Journal of Early Childhood Research, 13(2), 137-149, doi:10.1177/1476718x13490297

Lindquist, K. A., MacCormack, J. K., y Shablack, H. (2015). The role of language in emotion: Predictions from psychological constructionism. Frontiers in Psychology, 6, 1-17, doi:10.3389/fpsyg.2015.00444

López, E. (2005). La Educación emocional en la educación infantil. Revista Interuniversitaria de formación del profesorado, 19(3), 153-167.

McGlinchey, J. B., Atkins, D. C., y Jacobson, N. S. (2002). Clinical significance methods: Which one to use and how useful are they? Behavior Therapy, 33, 529-550, doi: 10.1016/s00057894(02)80015-6

Martín, C. (2002). El uso de técnicas y dinámicas participativas en educación para la paz. De la resolución de conflictos al apoyo mutuo. Revista Cultura y Educación CyE, 14(4), 391-401, doi: $10.1174 / 113564002762700862$

Mayer J.D. y Salovey, P. (1997). “What is emotional intelligence?” En P. Salovey y D. Sluyter (Eds), Emotional Development and Emotional Intelligence: Implications for Educators (pp. 331). New York: Basic Books.

McMahon, S., Washburn, J., Felix, E. D., Yakin, J., y Childrey, G. (2000). Violence prevention: Program effects on urban preschool and kindergarten children. Applied and Prevention Psychology, 9(4), 271-281, doi:10.1016/S0962-1849(00)80004-9

Mestre, M.V., Samper, P., y Frías, M.D. (2002). Procesos cognitivos y emocionales predictores de la conducta prosocial y agresiva: la empatía como factor modulador. Psicothema, 14, 227232. 
Middel, B., y van Sonderen, E. (2002). Statistical significant change versus relevant or important change in (quasi) experimental design: some conceptual and methodological problems in estimating magnitude of intervention-related change in health services research. International Journal of Integrated Care, 2, e15. Recuperado de https://www.ncbi.nlm.nih.gov/pmc/articles/PMC1480399/

Middel, B., Stewart, R., Bouma, J., van Sonderen, E., y van den Heuvel, W. J. (2001). How to validate clinically important change in health-related functional status. Is the magnitude of the effect size consistently related to magnitude of change as indicated by a global question rating? Journal of Evaluation in Clinical Practice, 7(4):399-410.

Ministerio de Educación (2019). Diseño Curricular Básico Nacional de la Formación Inicial del Docente. Lima, Perú: autor

Monin, B., Pizarro, D. y Beer, J. (2007). Deciding vs reacting: Conceptions of moral judgment and the reason-affect debate. Review of general Psychology, 1(2), 99-11, doi:10.1037/10892680.11.2.99.

Montañés, M. (2009). Metodología y técnica participativa: Teoría y práctica de una estrategia de investigación participativa. Barcelona, España: UOC.

Moral, J. Gonzáles, MT. y Landero, R. (2011). Estrés percibido, ira y burnout en amas de casa mexicanas. Revista Iberoamericana de Psicología y Salud, 2(12), 123-143.

Muthén, B. O. (1984). A general structural equation model with dichotomous, ordered categorical, and continuous latent variable indicators. Psychometrika, 49(1), 115-132, doi:10.1007/bf02294210

Muthén, B., du Toit, S.H.C. y Spisic, D. (1997). Robust inference using weighted least squares and quadratic estimating equations in latent variable modeling with categorical and continuous outcomes. Unpublished technical report. Recuperado de https://www.statmodel.com/download/Article_075.pdf (24 agosto 2009)

Muthén, L.K., y Muthén, B.O. (1998-2012). Mplus User's Guide (version 7) [Computer program]. Los Angeles, CA: Muthén y Muthén.

Obiols, M. (2005). Diseño, desarrollo y evaluación de un programa de educación emocional en un centro educativo. Revista Interuniversitaria de Formación de Profesorado, 19(3), 137-152.

Oblitas, L. (2008). El estado del arte de la psicología de la Salud. Revista de Psicología, 26(2), 219-254.

Oros, L.B. (2014). Nuevo cuestionario de emociones positivas para niños/as. Anales de Psicología, 30(2), 522-529, doi:10.6018/analesps.30.2.158361

Paul, S. (2006). Exploring the psychology of interest. New York: Oxford University Press

Pérez, Y. y Guerra, V.M. (2014). La regulación emocional y su implicación en la salud del adolescente. Revista Cubana de Pediatría, 86(3), 368-375.

Pichardo, M.C., García, T., Justicia, F y Llanos, C. (2008). Efectos de un programa de intervención para la mejora de la competencia social en niños/as de educación primaria en Bolivia. International Journal of Psychology and Psychological Therapy, 8(3), 441-452.

Piqueras, S.A., Ramos, V., Martínez, A. y Oblitas, L. (2009). Emociones negativas y su impacto en la salud mental y física. Suma Psicológica, 16(2), 85-112, doi:10.14349/sumapsi2009.136

Plonsky, L., y Oswald, F. L. (2014). How big is "big"? Interpreting effect sizes in L2 research. 
Language Learning, 64,878-912, doi:10.1111/lang.12079

Pulido, F., y Herrera, F. (2015). Miedo e inteligencia emocional en el contexto pluricultural de Ceuta. Anuario de Psicología, 45(2), 249-263

Pulido, F. y Herrera, F. (2017). La influencia de las emociones sobre el rendimiento académico. Ciencias Psicológicas, 11(1), 29-39, doi:10.22235/cp.v11i2.1344

Kushnir, J., Gothelf, D. y Sadeh, A. (2014). Nighttime fears of preschool children: A potential disposition marker for anxiety? Comprehensive Psychiatry, 55, 336-341, doi:10.1016/j.comppsych.2013.08.019

Raykov, T. (2012). Scale construction and development using structural equation modeling. In R. H. Hoyle (Ed.), Handbook of structural equation modeling (pp. 472-492). New York: Guildford Press.

Rendón, M.I. (2007). Regulación emocional y competencia social en la infancia. Diversitas. Perspectivas en Psicología, 3(2), 349-64.

Ronk, F. R., Hooke, G. R., y Page, A. C. (2016). Validity of clinically significant change classifications yielded by Jacobson-Truax and Hageman-Arrindell methods. BMC Psychiatry, 16, 187, doi:10.1186/s12888-016-0895-5

Ronk, F. R.., Hooke, G. R., y Page, A. C. (2012). How consistent are clinical significance classifications when calculation methods and outcome measures differ? Clinical Psychology: Science and Practice, 19(2), 167-179, doi:10.1111/j.14682850.2012.01281.x

Rueda, M. (2017). Reconocimiento emocional a partir de expresiones faciales y piezas musicales en niños/as y adultos. Tesis doctoral Universidad Autónoma de Madrid. Facultad de Psicología

Saavedra, J.S., Díaz, W.J., Zúñiga, L.F. y Zamora, T.O. (2015). Correlación funcional del sistema límbico con la emoción, el aprendizaje y la memoria. Morfolia, 7(2), 29-44.

Seco, M.S. (2006). Cuerpo de Maestros. Volumen Práctico. Educación Infantil. Sevilla: MAD.

Tarnowski, P., Kołodziej, M., Majkowski, A., y Rak, R. J. (2017). Emotion recognition using facial expressions. Procedia Computer Science, 108, 1175-1184, doi:10.1016/j.procs.2017.05.025

Tracy, J. L., y Robins, R. W. (2006). Appraisal antecedents of shame and guilt: Support for a theoretical model. Personality and Social Psychology Bulletin, 32, 1339-1351, doi: $10.1177 / 0146167206290212$

Tugade, M. y Fredrickson, B.L. (2007). Regulation of positive emotions: emotion regulation strategies that promote resilience. Journal of Happiness Studies, 8, 311-333, doi: $10.1007 / \mathrm{s} 10902-006-9015-4$

Turan, N. y Cohen, T.R. (2016). Shame and Guilt. Encyclopedia of Mental Health. Editor Howard Friedman $\quad(144-146)$. health/friedman/978-0-12-397045-9

Vecina, M.L. (2006). Emociones Positivas. Papeles del Psicólogo, 27(1). 9-17.

Wise, E. A. (2004). Methods for analyzing psychotherapy outcomes: A review of clinical significance, reliable change, and recommendations for future directions. Journal of Personality Assessment, 82, 50-59, doi:10.1207/s15327752jpa8201_10. 
Fecha de entrada: 07 julio 2019 Fecha de revisión: 29 febrero 2020 Fecha de aceptación: 07 marzo 2020 